\title{
Expression of renin-angiotensin system (RAS) components in endometrial cancer
}

\author{
Sarah J Delforce ${ }^{1,2,3}$, Eugenie R Lumbers ${ }^{1,2,3}$, Celine Corbisier de Meaultsart ${ }^{1,2,3}$,

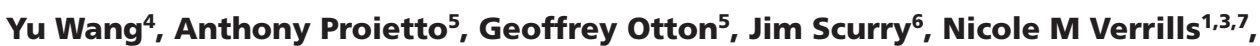 \\ Rodney J Scott ${ }^{1,3,6}$ and Kirsty G Pringle ${ }^{1,2,3}$
}

${ }^{1}$ School of Biomedical Sciences and Pharmacy, University of Newcastle, Newcastle, New South Wales, Australia ${ }^{2}$ Priority Research Centre for Reproductive Sciences, University of Newcastle, Newcastle, New South Wales, Australia

${ }^{3}$ Hunter Medical Research Institute, Newcastle, New South Wales, Australia

${ }^{4}$ Oregon Health and Science University, Portland, Oregon, USA

${ }^{5}$ Hunter Centre for Gynaecological Cancer, John Hunter Hospital, Newcastle, New South Wales, Australia

${ }^{6}$ Hunter Area Pathology Service, John Hunter Hospital, Newcastle, New South Wales, Australia

${ }^{7}$ Priority Research Centre for Cancer, University of Newcastle, Newcastle, New South Wales, Australia

Correspondence

should be addressed

to $\mathrm{K}$ G Pringle

Email

kirsty.pringle@newcastle.

edu.au

\section{Abstract}

A dysfunctional endometrial renin-angiotensin system (RAS) could aid the growth and spread of endometrial cancer. To determine if the RAS is altered in endometrial cancer, we measured RAS gene expression and protein levels in 30 human formalin-fixed, paraffin-embedded (FFPE) endometrioid carcinomas and their adjacent endometrium. All components of the RAS were expressed in most tumours and in adjacent endometrium; mRNA levels of (pro)renin receptor (ATP6AP2), angiotensin II type 1 receptor (AGTR1), angiotensin-converting enzyme (ACE1) and angiotensin-converting enzyme 2 ( $A C E 2$ ) mRNA levels were greater in tumour tissue than adjacent non-cancerous endometrium ( $P=0.023,0.008,0.004$ and 0.046 , respectively). Prorenin, ATP6AP2, AGTR1, AGTR2 and ACE2 proteins were abundantly expressed in both cancerous and adjacent non-cancerous endometrium. Staining was most intense in cancerous glandular epithelium. One potential target of the endometrial RAS, transforming growth factor beta-1 (TGFB1), which is essential for epithelial-to-mesenchymal transition, was also upregulated in endometrial cancer tissue $(P=0.001)$. Interestingly, TGFB1 was strongly correlated with RAS expression and was upregulated in tumour tissue. This study is the first to characterise the mRNA and protein expression of all RAS components in cancerous and adjacent non-cancerous endometrium. The greater expression of ATP6AP2, AGTR1 and $A C E 1$, key elements of the pro-angiogenic/proliferative arm of the RAS, suggests that the RAS plays a role in the growth and spread of endometrial cancer. Therefore, existing drugs that inhibit the RAS and which are used to treat hypertension may have potential as treatments for endometrial cancer.

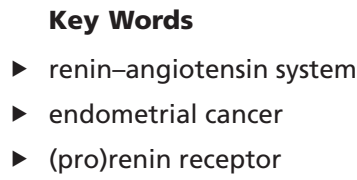

Endocrine Connections (2017) 6, 9-19

\section{Introduction}

Endometrial cancer is the most common gynaecological malignancy, and the sixth most common cancer in women worldwide, with 320,000 new cases and 76,000 deaths in 2012 (1). The incidence of endometrial cancer is increasing due to the ageing population and rising obesity levels. In over $50 \%$ of patients, endometrial cancer is associated with obesity with the risk increasing linearly with body mass index (BMI) $(2,3)$.
(C) 2017 The authors
Published by Bioscientifica Ltd

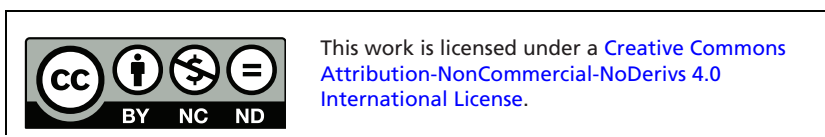


Healthy human endometrium from women of reproductive age, expresses all of the components of the renin-angiotensin system (RAS) $(4,5)$, including the prorenin receptor, which is also expressed in the endometrium during pregnancy (6). As this system is responsible for stimulating angiogenesis, cell proliferation and migration in the normal endometrium, it has the potential, if overexpressed and/or activated, to promote abnormal cell growth and spread that is the hallmark of endometrial cancer (Fig. 1). The prorenin receptor ((P)RR) is an integral component of a vacuolar (v)-ATPase that can acidify the extracellular milieu. The (P)RR also binds both prorenin and renin (7). Prorenin bound to the (P)RR can cleave angiotensin I (Ang I) from angiotensinogen (AGT). Angiotensin II (Ang II), cleaved from Ang I by angiotensinconverting enzyme (ACE), can act on the angiotensin II type 1 receptor (AGTR1) to stimulate angiogenesis and cell proliferation. There is also an additional RAS pathway that opposes the Ang II/AGTR1 pathway. This is the ACE2/Ang(1-7)/MasR pathway (Fig. 1). Prorenin binding to the $(\mathrm{P}) \mathrm{RR}$ also elicits intracellular signalling that is independent of Ang production and which is also proliferative and potentially tumourigenic (7).

We have already shown that the prevalence of a SNP (rs5186) in the AGTR1 gene, which is known to be associated with the overexpression of AGTR1 (8), is higher in women with endometrial cancer (9). Two other studies have shown that levels of expression of components of the
RAS change according to the clinicopathological features of endometrial cancer. Piastowska-Ciesielska and coworkers. showed that levels of expression of AGTR1, AGTR2, vascular endothelial growth factor (VEGF) and oestrogen receptor (ER)- $\alpha$ genes and proteins varied with the grade of cancer where both Ang II receptors were higher in earlygrade cancers (10). Furthermore, Shibata and coworkers demonstrated that levels of Ang II, AGTR1 and VEGF peptides and proteins as well as adipocyte-derived leucine aminopeptidase (A-LAP), which hydrolyses Ang II (11), were prognostic, with increased expression of A-LAP, which degrades Ang II, predicting better outcomes. However, there are other pathways by which the endometrial RAS could stimulate cancer growth. Neither (P)RR nor the ACE2/Ang(1-7)/MasR pathways have been examined in endometrial cancers.

Our aim was to measure the expression of genes and proteins (by immunohistochemistry) of the RAS pathways in endometrial cancer tissue and adjacent non-cancerous endometrium and to determine if the expression of any putative downstream targets of this pathway such as VEGFA, plasminogen activator inhibitor-1 (SERPINE1), transforming growth factor beta 1 (TGFB1) and phosphoinositide-3-kinase (PIK3R1) are expressed and if their levels of expression correlate with the expression of components of the (P)RR/prorenin/angiotensin system. We also examined the expression of cathepsin D (CTSD), a protease that is known to activate prorenin in vitro (12).

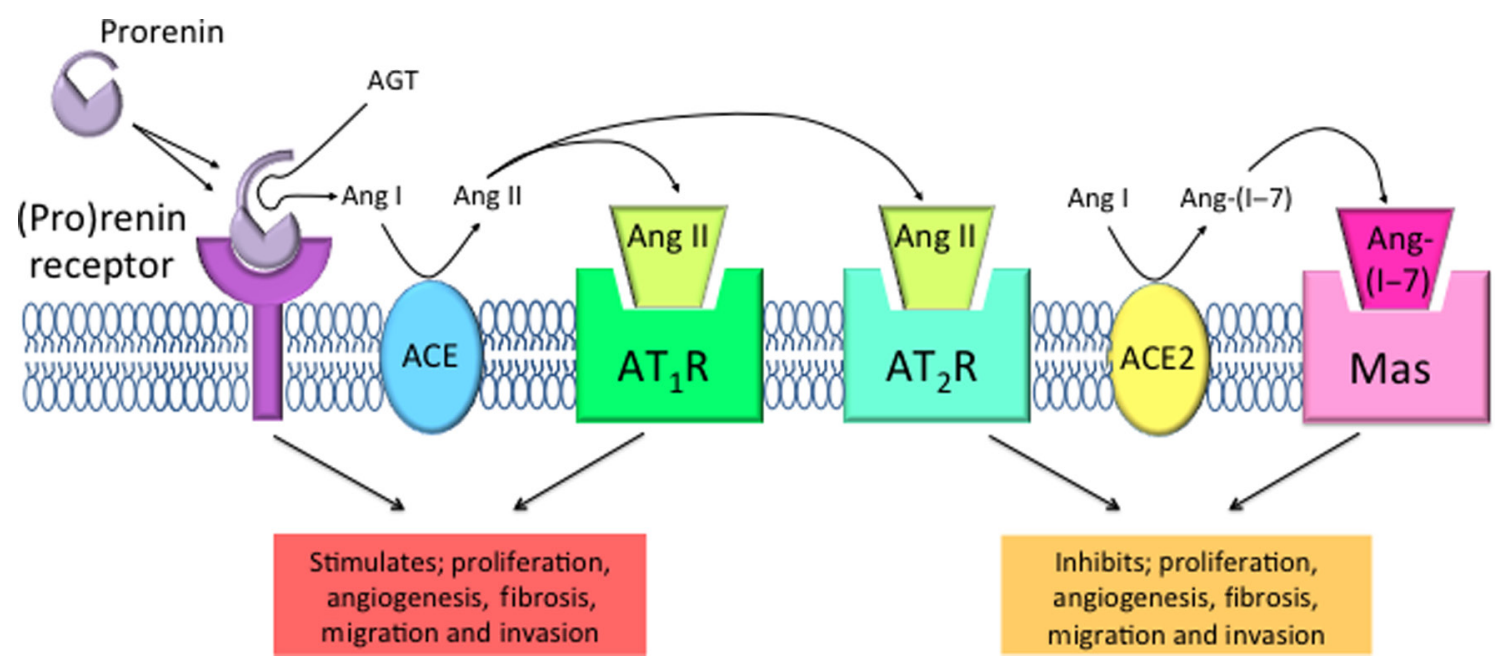

Figure 1

Tissue renin-angiotensin system cascade. Prorenin is activated by the (pro)renin receptor ((P)RR) and possibly by proteolysis, to cleave angiotensin (Ang) I from angiotensinogen (AGT). Angiotensin-converting enzyme (ACE) then converts Ang I to the biologically active Ang II. Ang II can bind to angiotensin II type 1 receptor (AGTR1) to promote proliferation, angiogenesis, fibrosis, migration and invasion through stimulation of growth factors and intracellular signalling pathways. Furthermore, angiotensin (Ang) II binds to angiotensin II type 2 receptor (AGTR2) and antagonises AGTR1 activation. Ang I can also be further converted by angiotensin-converting enzyme 2 (ACE2) to Ang(1-7). Ang(1-7) acts upon its receptor Mas. This results in antagonism of Ang II/AGTR1 stimulation, thus inhibiting proliferation, angiogenesis, fibrosis, migration and invasion.

http://www.endocrineconnections.org DOI: 10.1530/EC-16-0082 (c) 2017 The authors Published by Bioscientifica Ltd
This work is licensed under a Creative Commons Attribution-NonCommercial-NoDerivs 4.0 International License. 


\section{Methods}

\section{Endometrial cancer tissue sample collection}

This research was approved by the Hunter Area Research Ethics Committee and the University of Newcastle Human Research Ethics Committee. Thirty formalinfixed paraffin-embedded (FFPE) endometrial tumours and matched non-cancerous adjacent tissues from women with endometrioid adenocarcinomas were provided by the Hunter Cancer Biobank (HCB, John Hunter Hospital, Newcastle, Australia). Samples were stained with H\&E and the type, grade and percent of section that was tumour or benign tissue for each section determined by a pathologist. For this study, we examined $n=11$ of each Grade 1 and 2 and $n=8$ Grade 3 tumours. No exclusion criteria were used, as linked data were unavailable.

\section{Semi-quantitative real-time reverse transcriptase polymerase chain reaction (qPCR)}

Total RNA was isolated from FFPE tissues using the Qiagen RNeasy FFPE Kit (Qiagen) according to the manufacturer's instructions. Total RNA was spiked with a known amount of Alien reference RNA (Stratagene; $10^{7}$ copies per microgram of total RNA) before the RNA was reverse transcribed using a Superscript III RT kit with random hexamers (Invitrogen). The Alien qRT PCR inhibitor alert system serves as a reference for internal standardisation (13).

qPCR was performed in an Applied Biosystems 7500 Real-Time PCR System using SYBR Green for detection. Each reaction contained cDNA reverse transcribed from 10 ng total RNA, SYBR Green PCR master mix (Applied Biosystems) and primers. RAS primers used for the analysis of FFPE tissues were designed using OligoArchitect Online (v4.0; Sigma Aldrich) design tool for custom DNA probes for optimum use on FFPE samples (amplicon length of $<100 \mathrm{bp}$, listed in Table 1). Dissociation curves, to detect non-specific amplification, were generated for all reactions, and non-template control samples were included in all assays. The predicted sizes of the PCR products were verified by agarose gel electrophoresis (data not shown). mRNA abundance was constructed using a standard curve of the calibrator sample (a term placental sample collected at elective Caesarean section), and abundance was expressed relative to both Alien and $\beta$-actin $(A C T B)$ mRNA.

\section{Immunohistochemistry}

Five micron thick FFPE sections affixed to slides were de-waxed and antigen retrieval for prorenin, ATP6AP2,

Table 1 Primers and real-time PCR conditions used for the genes analysed.

\begin{tabular}{|c|c|c|c|c|}
\hline Official gene symbol & GenBank accession \# & Primer sequence $\left(5^{\prime} \rightarrow 3^{\prime}\right)$ & $\begin{array}{c}\text { Concentration } \\
\text { (nM) }\end{array}$ & $\begin{array}{l}\text { Detection temperature } \\
\qquad\left({ }^{\circ} \mathrm{C}\right)\end{array}$ \\
\hline \multirow[t]{2}{*}{$A C E 1$} & NM_000789 & Fwd: CAGGTGGTGTGGAACGAGTATGC & 200 & 77 \\
\hline & & Rev: TCTCTGTGGTGATGTTGGTGTTGTAGT & & \\
\hline \multirow[t]{2}{*}{ ACE2 } & NM_021804 & Fwd: AAGCACTCACGATTGTTGGGACTCT & 200 & 75 \\
\hline & & Rev: AAGACCATCCACСТCСАСТTСТСТАAC & & \\
\hline \multirow[t]{2}{*}{ ACTB } & NM_001101 & Fwd: CGCGAGAAGATGACCCAGAT & 1000 & 78 \\
\hline & & Rev: GAGTCCATCACGATGCCAGT & & \\
\hline \multirow[t]{2}{*}{$A G T$} & NM_000029 & Fwd: CGCCTGCCTGCTGCTGAT & 100 & 80 \\
\hline & & Rev: GGAAAGTGAGACCСТCСАССTTGT & & \\
\hline \multirow[t]{2}{*}{ AGTR1 } & NM_000685 & Fwd: GCCTCCTCGCCAATGATTCCA & 100 & 82 \\
\hline & & Rev: CGTCCTGTCACTCGCTGCTG & & \\
\hline \multirow[t]{2}{*}{ ATP6AP2 } & NM_005765 & Fwd: ACAATGAAGTTGACCTGCTCTTTCTTTCTG & 100 & 76 \\
\hline & & Rev: CCTTGGCTAGATGCTTATGACGAGACA & & \\
\hline \multirow{2}{*}{ PIK3R1 } & NM_181523 & Fwd: GAGGGAAGCGAGATGGCACTTT & 200 & 80 \\
\hline & & Rev: TCCACCACTACAGAGCAGGCATA & & \\
\hline \multirow[t]{2}{*}{$R E N$} & NM_000537 & Fwd: CCACCTCCTCCGTGATCCT & 200 & 76 \\
\hline & & Rev: GCGGATAGTACTGGGTGTCCAT & & \\
\hline \multirow[t]{2}{*}{ SERPINE1 } & NM_000602.4 & Fwd: TCTGTGTCACCGTATCTCA & 200 & 80 \\
\hline & & Rev: GCTCCGTCACGCTGGATGTC & & \\
\hline \multirow[t]{2}{*}{ TGFB1 } & NM_000660 & Fwd: GAACTCATTCAGTCACCATAGCAACACTCT & 400 & 76 \\
\hline & & Rev: TCTCTGGGCTTGTTTCCTCACCTTTA & & \\
\hline \multirow[t]{2}{*}{ VEGFA } & M32977 & Fwd: CTACCTCCACCATGCCAAGT & 400 & 82 \\
\hline & & Rev: GCAGTAGCTGCGCTGATAGA & & \\
\hline
\end{tabular}

Fwd: forward primer; Rev: reverse primer.

http://www.endocrineconnections.org DOI: 10.1530/EC-16-0082
C) 2017 The authors Published by Bioscientifica Ltd

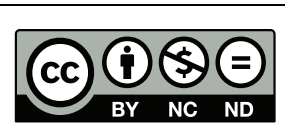

This work is licensed under a Creative Commons Attribution-NonCommercial-NoDerivs 4.0 International License. 
ACE1, ACE2, AGT, AGTR1 and MAS1 was performed in $10 \mathrm{mM}$ citrate buffer at $\mathrm{pH} 6.0$ for $10 \mathrm{~min}$ using a microwave oven. Antigen retrieval for AGTR2 was performed in a hybridisation oven with Proteinase K (1:1000) for $10 \mathrm{~min}$ at $37^{\circ} \mathrm{C}$ for $10 \mathrm{~min}$. Antibodies used were ACE1 (BosterBio, PA2196-1, $2.5 \mu \mathrm{g} / \mathrm{mL}$ ), ACE2 (Abcam, ab15348, $0.005 \mathrm{mg} / \mathrm{mL}$ ), AGT (R\&D Systems, af3156, $0.002 \mathrm{mg} / \mathrm{mL}$ ), AGTR1 (Abcam, ab9391, 0.125 mg/mL), AGTR2 (Abcam, ab19134, 0.012 mg/mL), MAS1 (Abcam, ab140854, $0.1 \mathrm{mg} / \mathrm{mL}$ ), ATP6AP2 (Everest Biotech, eb06118, $10 \mu \mathrm{g} / \mathrm{mL}$ ) and REN propeptide (R\&D Systems, MAB4447, $0.05 \mathrm{mg} / \mathrm{mL}$ ). The positive control tissue was a first trimester placenta sample collected at elective termination of pregnancy. Matched samples without the addition of primary antibody were included as negative controls. Sections were blocked with bovine serum albumin (BSA) blocking solution $(0.5 \% \mathrm{BSA} w / \mathrm{v}, 0.05 \% \mathrm{w} / \mathrm{v}$ Saponin in $0.1 \mathrm{M}$ PBS) for $1 \mathrm{~h}$ at room temperature and then incubated overnight with primary antibody. Images were captured using the Aperio AT Turbo slide scanner (Leica Biosystems).

\section{Statistical analyses}

mRNA data were analysed using Graphpad Prism, version 6.0. The relationships between tumour grade and mRNA abundance were determined using non-parametric Kruskal-Wallis tests. Wilcoxon matched-pairs signedrank tests were used to identify differences in mRNA abundance between tumour and matched adjacent noncancerous endometrium. To determine the associations between particular RAS genes and putative downstream targets, Spearman's non-parametric correlations were used. If a sample had undetectable levels of mRNA, a value of $1 \times 10^{-5}$ was allocated so that it could be included in the analyses. Significance was set at $P<0.05$ for all data.

\section{Results}

\section{Expression of renin-angiotensin system components and their downstream targets is not associated with endometrial tumour grade}

The histopathological classification of tumour grade was not associated with the level of expression of any of the genes studied. The expression of VEGFA tended to increase with tumour grade; however, this failed to reach significance ( $P=0.063, n=30$, data not shown). As mRNA abundance was not affected by cancer grade, data were pooled for subsequent analyses.

$$
\begin{aligned}
& \text { http://www.endocrineconnections.org } \\
& \text { DOI: 10.1530/EC-16-0082 }
\end{aligned}
$$

\section{Expression of prorenin and the (pro)renin receptor in endometrial cancer and adjacent non-cancerous endometrial samples}

All tumours expressed REN mRNA as did all but 2 adjacent non-cancerous tissues, whereas prorenin protein was abundantly expressed in all samples (Fig. 2). There was no difference in the levels of expression of REN between tumours and the adjacent endometrium. The pervasive prorenin immunostaining throughout the glandular and stromal tissue compartments of both tumour and noncancerous adjacent endometrium suggests that levels of expression of REN in cancerous and non-cancerous endometrium were comparable.

The (pro)renin receptor gene (ATP6AP2) was expressed in 27/30 tumour samples and in their matched endometrium and its expression was significantly greater in cancer tissue compared with adjacent non-cancerous endometrium $(P=0.02$, Fig. 2). Immunostaining of prorenin and ATP6AP2 was most intense in the glandular epithelium (Fig. 2). ATP6AP2 protein was also present in the stroma but was localised to the perivascular space and endothelium.

\section{Expression of cathepsin D mRNA in endometrial cancer and adjacent non-cancerous endometrial samples}

To determine the potential for cathepsin $\mathrm{D}$ to proteolytically activate prorenin within an acidic milieu created through the hypoxic environment of the tumour, which can be generated by the v-ATPase activity of (P)RR, we measured mRNA levels of expression of cathepsin D. CTSD mRNA levels tended to be greater in tumour tissue than in adjacent non-cancerous endometrium $(P=0.059$, Fig. 3$)$.

\section{Expression of renin-angiotensin system genes in cancerous and adjacent non-cancerous endometrial samples}

AGT, AGTR1, ACE1 and ACE2 were detected in 28/30, 26/30, 23/30 and 25/30 cancers, respectively (Fig. 4). Expression of these genes was detected in the noncancerous adjacent tissue of 23/30, 24/30, 23/30 and $22 / 30$ cases. The protein products of all genes were detected by immunohistochemistry in all endometrial tumours and non-cancerous adjacent tissue (Fig. 5). Two Grade 3 tumours, which expressed REN abundantly as did

This work is licensed under a Creative Commons Attribution-NonCommercial-NoDerivs 4.0 International License. 

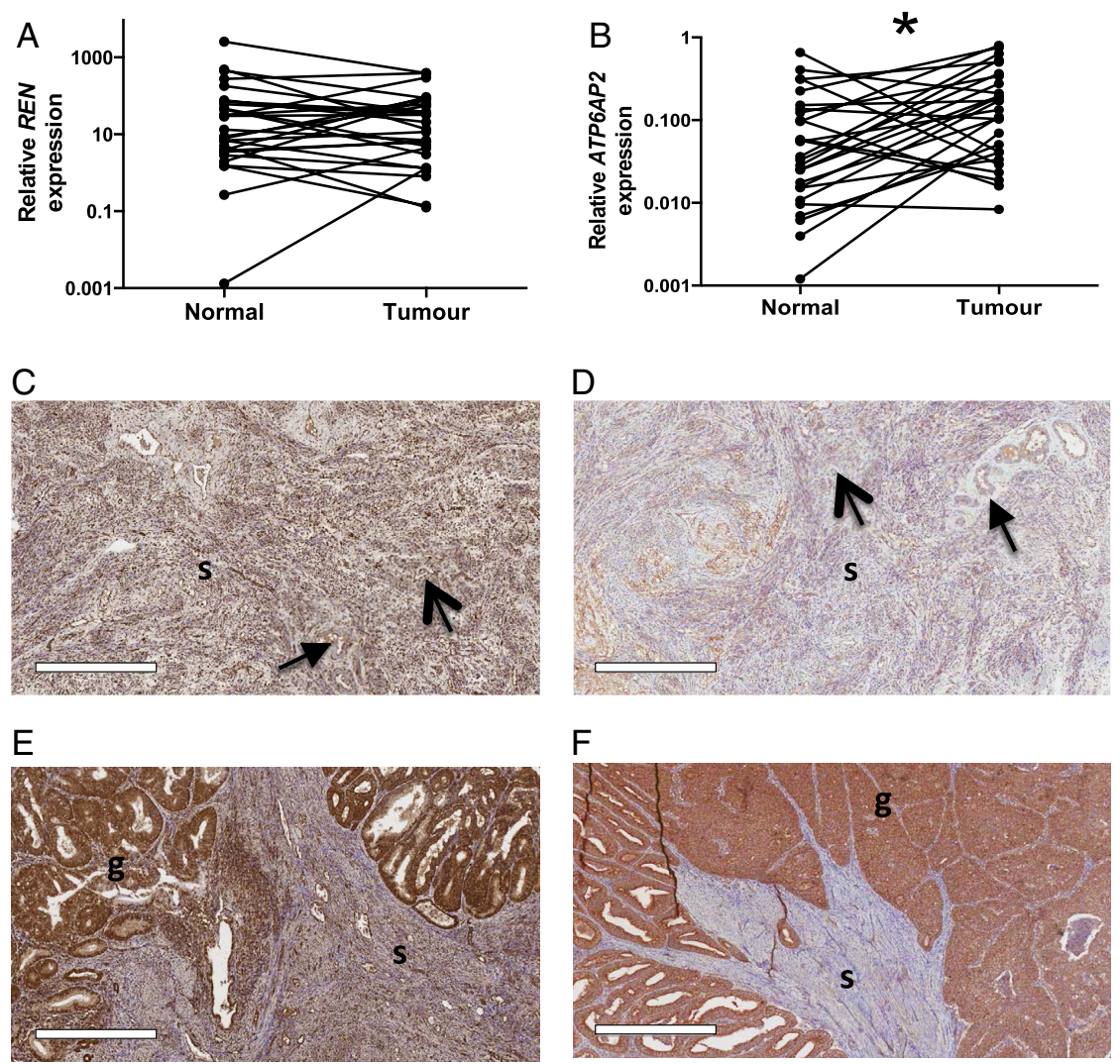

\section{Figure 2}

Prorenin and (P)RR mRNA and protein expression in endometrial cancer and matched adjacent non-cancerous endometrium. (A) There was no difference in REN mRNA expression between tumour and adjacent non-cancerous endometrium. (B) ATP6AP2 mRNA expression was greater in tumour ( ${ }^{*} P=0.023$ ) than that in adjacent endometrium. Scale bars $=500 \mu \mathrm{m}$. Images in the bottom panels were taken at a higher magnification, scale bars $=200 \mu \mathrm{m}$. Prorenin immunostaining was intense in both glandular (g) and stromal (s) tissue of both the

$\mathrm{H}$
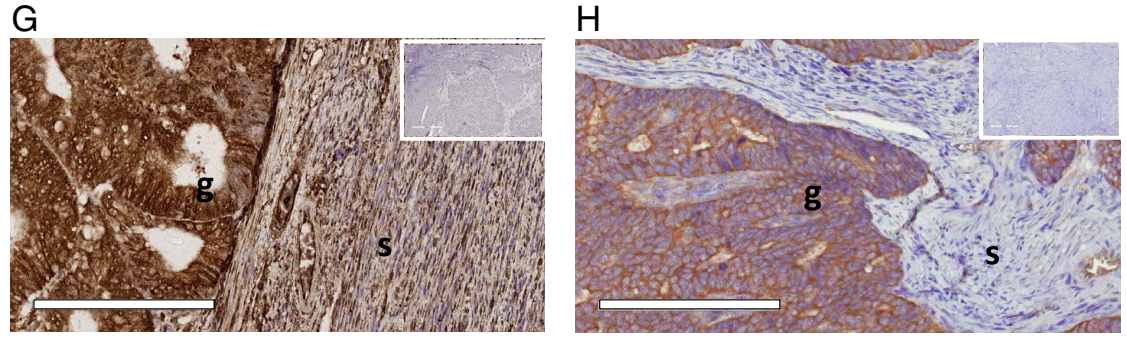
adjacent non-cancerous endometrium (C) and tumour ( $E$ and G). ATP6AP2, although intensely expressed in tumour glandular epithelium ( $\mathrm{F}$ and $\mathrm{H})$, was localised to the perivascular space (closed head arrow) and endothelium (open head arrow) in the adjacent normal endometrium (E). Immunostaining was not seen in the negative controls containing no primary antibody (inserts, tumour tissues).

their adjacent endometrium, had no detectable levels of other RAS genes.

There was no difference in $A G T$ mRNA between cancerous and non-cancerous adjacent tissue. In contrast, ACE1, AGTR1 and ACE2 mRNAs were significantly

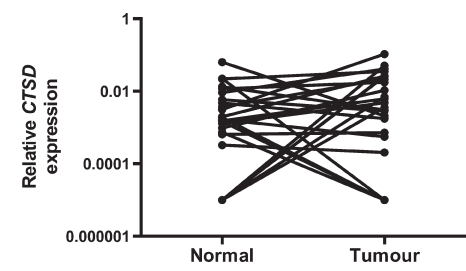

Figure 3

CTSD mRNA expression in tumour and matched adjacent non-cancerous endometrium. CTSD tended to be higher in tumour tissue when compared with matched adjacent non-cancerous endometrium ( $P=0.058)$. CTSD has the potential to activate prorenin in a low pH milieu (12). greater in tumour tissue compared with non-cancerous adjacent tissue $(P=0.004,0.008$ and 0.046 , respectively, Fig. 4). Immunostaining of AGT, ACE1, ACE2, AGTR1, AGTR2 and MAS1 protein was most intense in the glandular epithelium; it was also present in the stroma but was localised to the perivascular space and endothelium (Fig. 5).

\section{Expression of downstream targets of the renin-angiotensin system in endometrial cancer and adjacent non-cancerous endometrial samples}

VEGFA and SERPINE1 mRNA levels were the same in cancerous and matched non-cancerous endometrial tissue (Fig. 6). PIK3R1 mRNA was higher in tumour compared

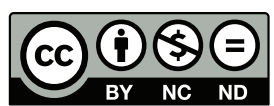

This work is licensed under a Creative Commons Attribution-NonCommercial-NoDerivs 4.0 International License. 

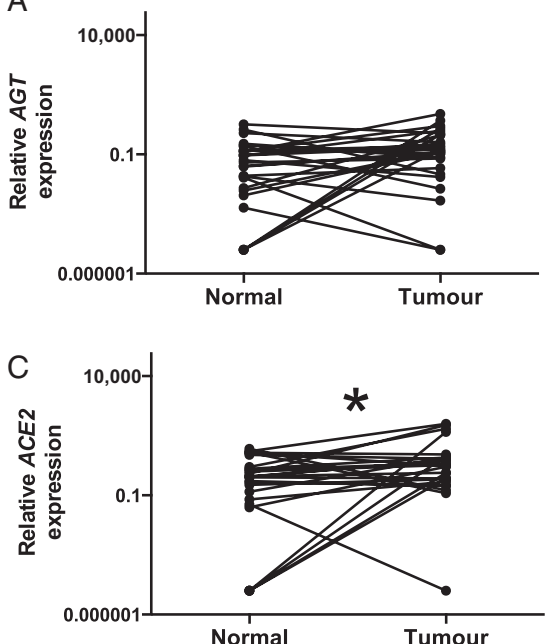

B
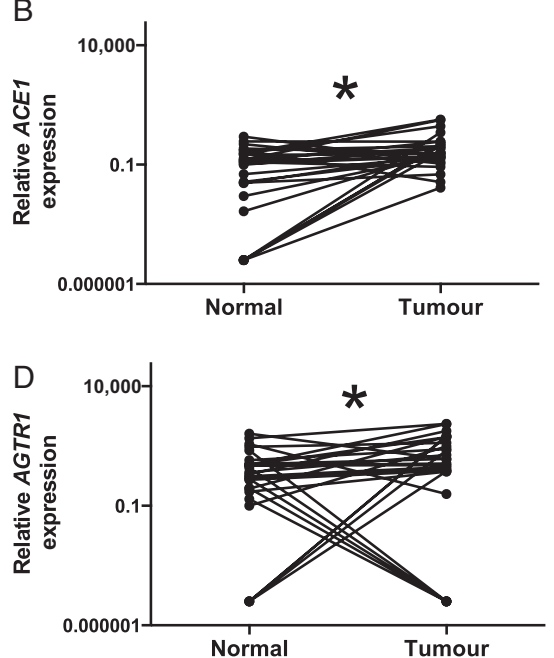

\section{Figure 4}

Expression of RAS mRNA levels in matched tumour and adjacent non-cancerous endometrium. AGT mRNA (A) was similar between tumour and adjacent non-cancerous endometria. ACE1 (B), ACE2 (C) and AGTR1 (D) mRNAs were all increased in tumour tissue compared with adjacent non-cancerous endometria $\left({ }^{*} P=0.004,0.046\right.$ and 0.008 , respectively). with matched non-cancerous tissue, although this failed to reach significance $(P=0.053$, Fig. 6). TGFB1 mRNA levels were significantly greater in tumour compared with non-cancerous adjacent tissue $(P=0.001$, Fig. 6).

As TGFB1, VEGFA, SERPINE1 and PIK3R1 are all potential targets of the $(\mathrm{P}) \mathrm{RR} /$ prorenin/Ang axis, we examined the association between the expression of components of the RAS pathway and these targets in both non-cancerous and cancerous endometrium. As shown in Table 2, in non-cancerous endometrium, REN mRNA correlated positively with the expression of both PIK3R1 and SERPINE1; no such correlations existed in tumour tissue. There were, however, direct positive correlations between the expression of the (pro)renin receptor (ATP6AP2) and all downstream targets in both non-cancerous and tumour tissue. Other components of the RAS were positively correlated with the expression of TGFB1 in both cancerous and non-cancerous tissue but were only correlated with VEGFA, CTSD, PIK3R1 and SERPINE1 in non-cancerous adjacent tissue.

\section{Discussion}

Most endometrial cancers and their non-cancerous adjacent tissues expressed the genes of the RAS, as well as that of its putative targets and other associated genes. We observed greater abundance of ATP6AP2, ACE1, AGTR1 and ACE2 mRNA in tumour tissue compared with their matched adjacent non-cancerous endometrium as assessed by mRNA analysis. These components are known to promote angiogenesis and tumourigenesis by both angiotensin-dependent and -independent pathways and thus could contribute to tumourigenesis (Fig. 7). Only $A G T$ and REN were not differentially expressed between tumour and non-cancerous tissue. There was no effect of tumour grade on mRNA levels. We have also shown that prorenin, ATP6AP2, AGT, ACE1, ACE2 and MAS1 proteins were present in both non-cancerous and tumour tissue as were both angiotensin receptor subtypes (AGTR1 and AGTR2). Staining for most of these proteins was most intense in the glandular epithelia of the tumour. As the endometrial cancers studied were adenocarcinomas, it was not surprising that expression of both genes and proteins were most abundant in the glandular tumour tissue compared with the adjacent noncancerous endometrium, which had very little, if any glandular epithelium. Stromal staining across all samples was weak and often confined to the perivascular space and endothelium.

The density of prorenin staining in adjacent noncancerous endometrium was intense although not as marked as in the glandular epithelium of the tumour. Even though prorenin was not differentially expressed between tumour and non-cancerous adjacent tissue, CTSD mRNA levels were significantly increased in tumour tissue. The increased abundance of CTSD in tumour tissue (Fig. 3), which is able to activate prorenin in a low $\mathrm{pH}$ milieu such as that created by the v-ATPase, of which the (P)RR is an integral component (7), may result in considerable amounts of Ang II being formed both in tumour and noncancerous adjacent tissue from AGT.

AGT protein was also highly expressed in the glandular epithelium, stroma, perivascular region and endothelium in endometrial cancer tissue (Fig. 4) and in adjacent non-cancerous endometrium. Ferguson and coworkers http://www.endocrineconnections.org DOI: 10.1530/EC-16-0082
() 2017 The authors Published by Bioscientifica Ltd
This work is licensed under a Creative Commons Attribution-NonCommercial-NoDerivs 4.0 International License. 

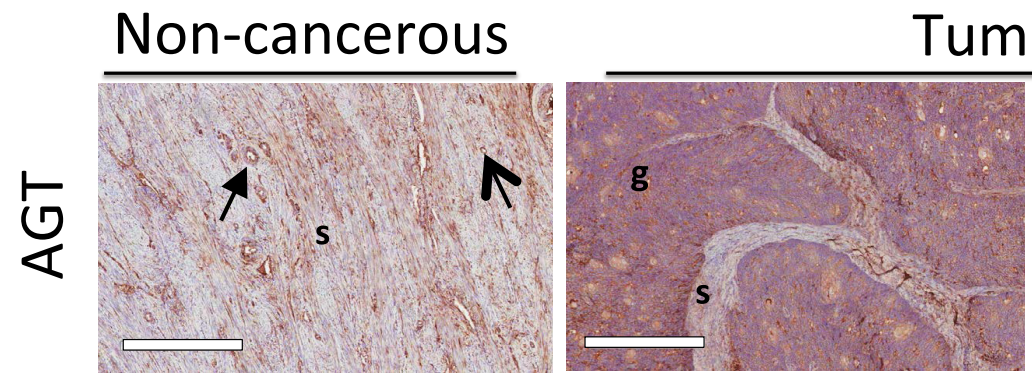

\section{Tumour}
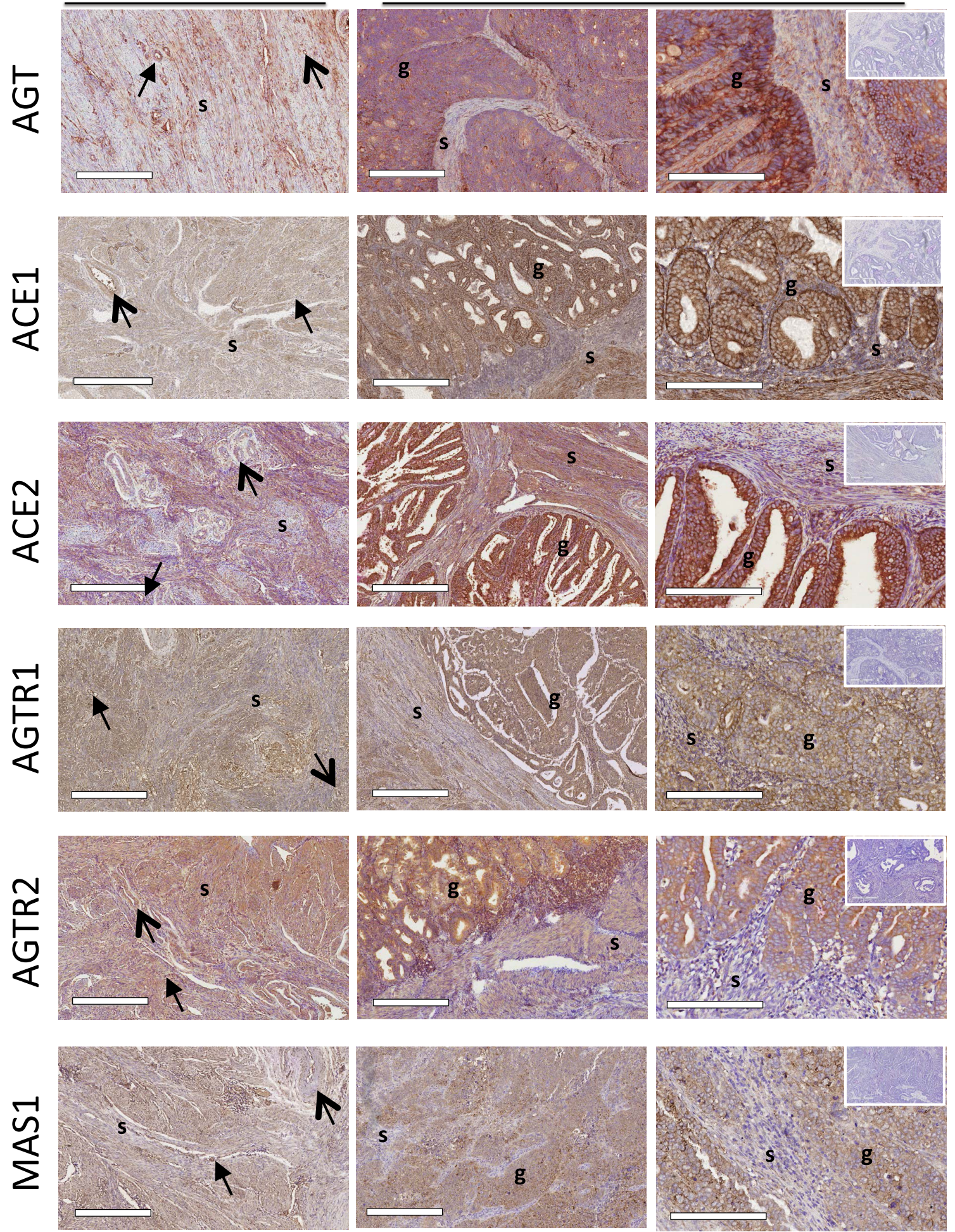

Figure 5

Localisation of RAS protein levels in matched tumour and adjacent non-cancerous endometrium. Scale bars $=500 \mu \mathrm{m}$. Images in the right hand panel were taken at a higher magnification, scale bars $=200 \mu \mathrm{m}$. Immunostaining of AGT, ACE1, ACE2, AGTR1, AGTR2 and MASR was present in the stroma (s) of both tumour and adjacent non-cancerous endometria and was localised to the perivascular space (closed head arrow) and endothelium (open head arrow). AGT, ACE1, ACE2, AGTR1, AGTR2 and MASR staining was most intense in the glandular epithelium (g) of the tumour. Immunostaining was not seen in the negative controls containing no primary antibody (inserts, tumour tissues).

\begin{tabular}{|lr}
\hline http://www.endocrineconnections.org & ○ 2017 The authors \\
DOI: $10.1530 /$ EC-16-0082 & Published by Bioscientifica Ltd
\end{tabular}


A

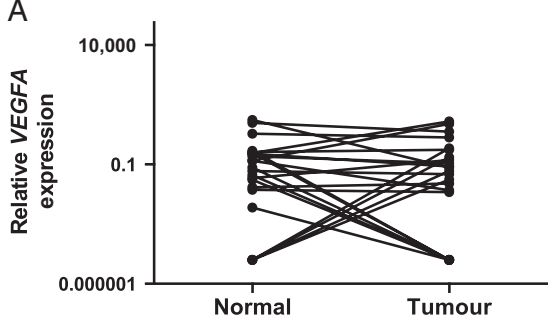

C

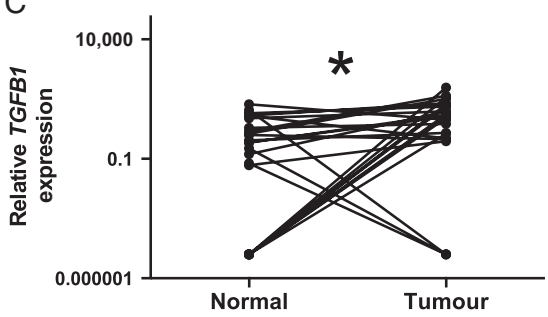

B

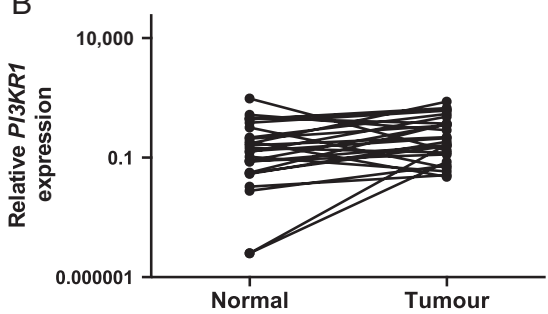

D

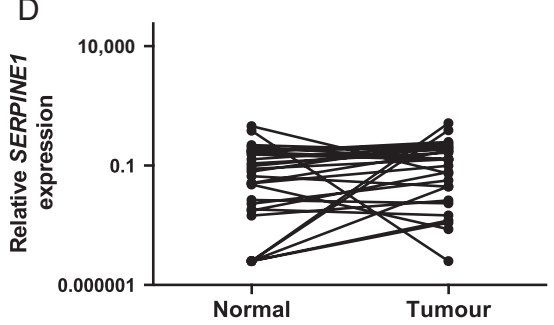

Figure 6

Expression of downstream targets of the RAS in matched tumour and adjacent non-cancerous endometrium. (A) VEGFA and (D) SERPINE1 mRNA levels were the same in tumour and matched adjacent non-cancerous endometrium. (B) PIK3R1 tended to be higher in tumour tissue $(P=0.053)$. (C) TGFB1 mRNA was significantly increased in tumour tissue when compared with matched adjacent non-cancerous endometrium $\left({ }^{*} P=0.001\right)$. determined that an increased expression of $A G T$ mRNA is associated with an increased risk of endometrial cancer recurrence (14). This suggests that AGT may contribute to tumour growth and progression via Ang II production and increased activation of its receptors. AGT production in the liver is oestrogen dependent (15). It is also expressed and secreted by adipose tissue (16). Both high oestrogen levels and obesity are major risk factors for endometrial cancer; therefore, their ability to increase AGT production may contribute to disease progression. High levels of ACE1 mRNA in endometrial tumours could also enhance their Ang II forming capacity. Lever and coworkers found that long-term use of ACE inhibitors for the treatment of hypertension decreased the risk of female-specific cancers in women (OR: 0.37 (0.12-0.87)) (17).

The current study also demonstrates that there is increased expression of AGTR1 mRNA in endometrial tumours and, as with other RAS proteins, this receptor appeared to be most highly expressed in the glandular epithelium. Recently, we have shown that women with endometrial cancer have a higher prevalence of the
SNP, rs5186 (A1166C in AGTR1) (9), which is associated with the upregulation of AGTR1 (8). Our finding that endometrial cancers express more AGTR1 mRNA reinforces the evidence that this SNP is a potential risk factor for endometrial cancer progression.

Shibata and coworkers found that higher levels of both Ang II and AGTR1 in endometrial cancer tissue predicted a poorer prognosis, whereas higher levels of adipocyte-derived leucine aminopeptidase (A-LAP), which degrades Ang II, predicted a better outcome (11). Our findings indicate that endometrial cancers have an increased capacity to produce Ang II and have increased AGTR1 expression. Thus, they support Shibata's conclusions. We have not measured AGTR2 mRNA levels, although like AGTR1 protein, AGTR2 protein was most dense in the glandular epithelium, stroma and perivascular space (Fig. 5). Our proposal that endometrial cancers may have an increased capacity to produce Ang II, as well as our observation of increased AGTR1 expression in the tumour tissue may help to explain Shibata and coworkers' observations that higher levels of both Ang II

Table 2 Spearman correlations between genes that control the activity of the RAS and targets and other associated genes.

\begin{tabular}{|c|c|c|}
\hline Target & & ATP6AP2 \\
\hline \multirow[t]{2}{*}{ VEGFA } & Tumour & $0.64(0.000)$ \\
\hline & Non-cancerous & $0.73(0.00)$ \\
\hline \multirow[t]{2}{*}{ PIK3R1 } & Tumour & $0.79(000)$ \\
\hline & Non-cancerous & $0.77(0.00)$ \\
\hline \multirow[t]{2}{*}{ SERPINE1 } & Tumour & $0.41(0.026)$ \\
\hline & Non-cancerous & $0.54(0.002)$ \\
\hline \multirow[t]{2}{*}{ TGFB1 } & Tumour & $0.76(000)$ \\
\hline & Non-cancerous & $0.68(0.000)$ \\
\hline
\end{tabular}

\begin{tabular}{c}
\hline REN \\
\hline- \\
- \\
- \\
$0.58(0.001)$ \\
- \\
$0.64(0.000)$ \\
- \\
-
\end{tabular}

\begin{tabular}{c}
\hline $\boldsymbol{A G T}$ \\
\hline $0.41(0.026)$ \\
- \\
- \\
- \\
- \\
- \\
$0.71(0.000)$
\end{tabular}

\begin{tabular}{c} 
ACE1 \\
\hline- \\
$0.58(0.000)$ \\
- \\
$0.5(0.005)$ \\
- \\
- \\
$0.68(000)$ \\
$0.61(000)$
\end{tabular}

\begin{tabular}{c} 
AGTR1 \\
\hline- \\
$0.37(0.04)$ \\
- \\
- \\
- \\
$0.41(0.02)$ \\
$0.43(0.019)$ \\
$0.47(0.009)$
\end{tabular}

\begin{tabular}{c}
\hline ACE2 \\
\hline- \\
$0.53(0.003)$ \\
- \\
$0.52(0.004)$ \\
- \\
- \\
$0.63(000)$ \\
$0.37(0.044)$
\end{tabular}

Spearman correlations ( $r$ ). Data expressed as $r$ (P value). All $n=30$, except $R E N$ where $n=28$.

http://www.endocrineconnections.org
DOI: $10.1530 /$ EC-16-0082 (c) 2017 The authors Published by Bioscientifica Ltd
This work is licensed under a Creative Commons Attribution-NonCommercial-NoDerivs 4.0 International License. 


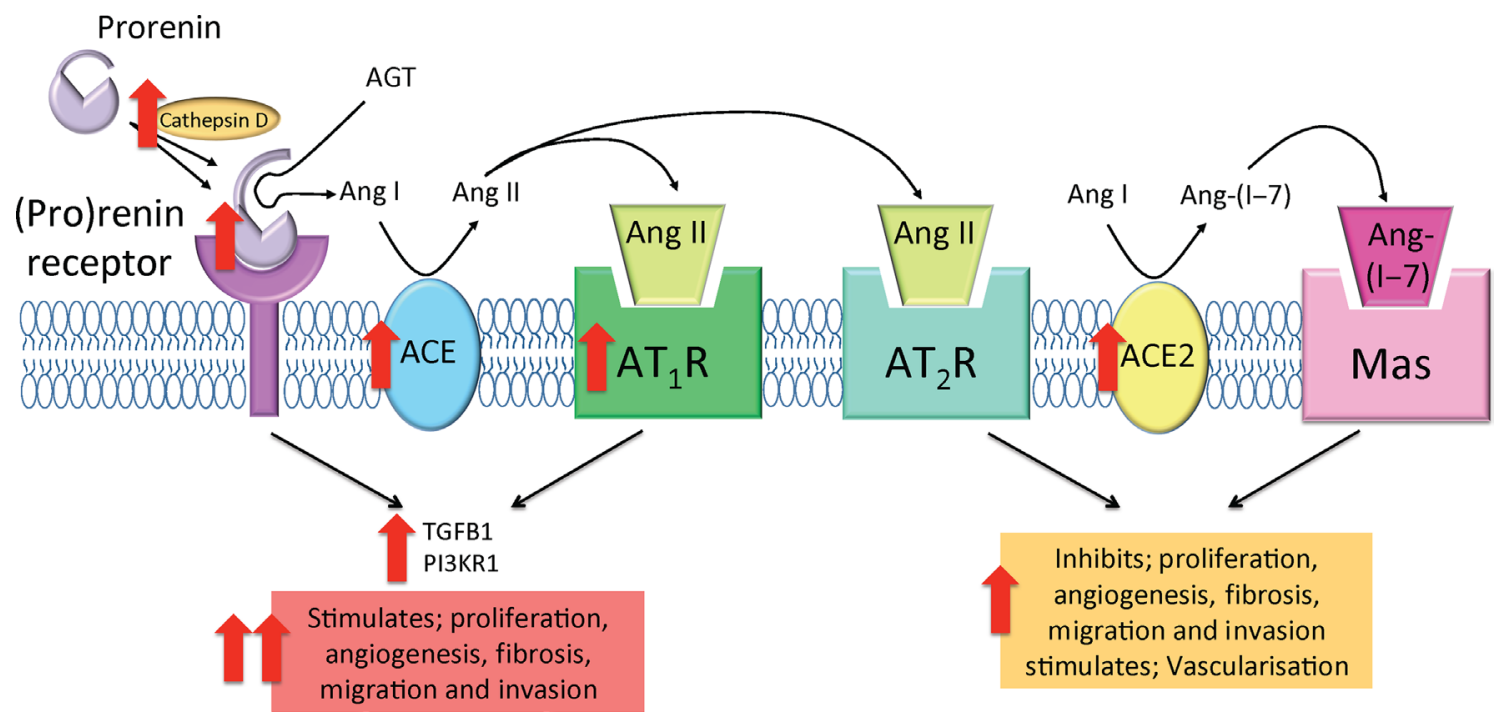

Figure 7

Tissue renin-angiotensin systems in endometrial cancer. In tumour tissue, upregulation of cathepsin $D$ and (P)RR allows for greater activation of prorenin, potentially leading to increased enzymatic cleavage of Ang I from AGT. Additionally, increased ACE1 abundance may lead to increased production of the biologically active Ang II. Together, upregulation of the pro-angiogenic/pro-proliferative components may lead to increased activation of both (P)RR- and AGTR1-mediated intracellular signalling cascades to stimulate the production of TGFB1 and PI3KR1 and promote tumourigenesis. The role of increased ACE2 and presence of MAS1 and AGTR2 may enhance tumour development by stimulating vascularisation; however, this is unknown.

and AGTR1 in endometrial cancer tissue predicted a poorer prognosis, whereas higher levels of adipocytederived leucine aminopeptidase (A-LAP), which degrades Ang II, predicted for a better overall outcome (11).

Although the role of ACE2, AGTR2 and MAS1 in the growth and spread of endometrial cancer is currently unknown, we have found that AGTR2 protein, like AGTR1 protein, was most intensely stained in the glandular epithelium, stroma and perivascular space (Fig. 5). We have also localised ACE2, AGTR2 and MAS1 proteins to the glandular epithelium, stroma, perivascular space and endothelium (Fig. 5). Although ACE2 mRNA abundance was significantly increased in endometrial cancer tissue when compared with normal adjacent endometrium (Fig. 4), we have not measured AGTR2 mRNA levels. Piastowska-Ciesielska and coworkers described a significant correlation between AGTR1 and AGTR2 mRNA expression in Grade 1 and Grade 2 tumours (10), indicating that although AGTR2 is not significantly higher in earlygrade tumours, AGTR1 and AGTR2 are associated. Given that AGTR1 is known to form a heterodimer with AGTR2 and MAS1 has been shown to form a hetero-oligomeric complex with AGTR1, both of which are able to dampen AGTR1-mediated signalling $(18,19)$, the increased ACE2/Ang(1-7)/MASR/AGTR2 pathway in endometrial cancer may act as a compensatory mechanism to counteract the actions of Ang II/AGTR1.
The increased activation of the RAS through both Ang II/AGTR1 and prorenin/(P)RR could contribute to tumourigenesis through stimulation of downstream factors that contribute to proliferation, angiogenesis, migration and invasion. Therefore, we attempted to explore the link between the RAS and four known downstream targets that may promote cancer growth and spread. Interestingly, expression of all four targets was strongly related to the expression of ATP6AP2 mRNA in both cancerous and adjacent non-cancerous endometria. This suggests that the (P)RR could play a role in both physiological and pathological endometrial growth and angiogenesis. However, in non-cancerous adjacent tissue, an association was found between a number of genes of the RAS and putative downstream targets (Table 2) that were not evident in tumour tissue. This may be due to other factors overriding the normal physiological regulation of these putative RAS pathway targets e.g. VEGFA expression was related to AGT, ACE, AGTR1 and ACE2 expression in noncancerous adjacent tissue but not in tumour tissue. VEGFA has been shown to be significantly higher in endometrial adenocarcinomas compared to normal (10). Furthermore, we have shown that in a breast cancer cell line (MCF-7), culture in low oxygen stimulates large amounts of VEGFA mRNA and protein in the absence of changes in REN or other RAS genes (Pringle KG, Wang Y, 
Scott R \& Lumbers ER, unpublished observations). We postulate that in the normal adjacent tissue, the RAS pathway induces the expression of growth factors that aid the spread of tumour tissue. However, this regulatory pathway is lost when the oxygen and $\mathrm{pH}$ levels of the tumour tissue become direct drivers of gene expression.

Interestingly, TGFB1, which is essential for epithelialto-mesenchymal transition of metastatic cells (20) and is regulated by the RAS, was strongly correlated with RAS expression and was upregulated in tumour tissue. Huang and coworkers showed that in mesangial cells, both renin and prorenin caused dose- and time-dependent increases in TGFB1 and SERPINE1 that were only inhibited by a (P)RR siRNA and not by an angiotensin inhibitor (21). The associations we have found in both non-cancerous and cancerous tissue between ATP6AP2 and TGFB1 and SERPINE1 support these findings. The strong associations between the expression of ATP6AP2 and both VEGFA and PIK3R1 in both non-cancerous and tumour tissue also suggest a dominant role for the (P)RR in determining the levels of production of these proteins. Furthermore, there were three tumours $(1 \times$ Grade 1 and $2 \times$ Grade 3$)$ in which the expression of ATP6AP2 was below the level of detection. None of these tumours expressed any of the targets listed in Table 2 except for one Grade 3 tumour, which did express SERPINE1. Taken together with the strong correlations with ATP6AP2 expression and target genes listed in Table 2, we further suggest that the activity of the (P)RR and its multiple signalling pathways has a major influence on the expression of the target genes we studied.

Patient information including hypertensive status, use of blood pressure medications including ACE inhibitors or AGTR1 blockers, BMI and diabetes were unavailable for the study. These factors could be potentially confounding and may contribute to the variability in the data. However, as the study used Wilcoxon matched-pairs signed-rank tests, the effects of confounders were reduced.

Overall, this study contributes to the emerging but exciting literature on the role of both canonical (Ang II/AGTR1) and non-canonical (Ang II-independent (P)RR signalling) RAS activation in tumourigenesis (Fig. 7). As such, the (P)RR has the potential to be an ideal target for the development of new anti-cancer therapies.

\section{Declaration of interest}

The authors declare that there is no conflict of interest that could be perceived as prejudicing the impartiality of the research reported.

\section{Funding}

This work was funded by the Hunter Medical Research Institute and the University of Newcastle. N M V is supported by a Cancer Institute NSW Fellowship. K G P is supported by an ARC Future Fellowship.

\section{Acknowledgements}

The authors would also like to acknowledge the Hunter Cancer Biobank and the Hunter Cancer Research Alliance for the kind donation of the endometrial cancer tissue samples.

\section{References}

1 Ferlay J, Soerjomataram I, Dikshit R, Eser S, Mathers C, Rebelo M, Parkin DM, Forman D \& Bray F 2015 Cancer incidence and mortality worldwide: sources, methods and major patterns in GLOBOCAN 2012. International Journal of Cancer 136 E359-E386. (doi:10.1002/ ijc.29210)

2 Lindemann K, Vatten LJ, Ellstrom-Engh M \& Eskild A 2008 Body mass, diabetes and smoking, and endometrial cancer risk: a follow-up study. British Journal of Cancer 98 1582-1585. (doi:10.1038/ sj.bjc.6604313)

3 McCullough ML, Patel AV, Patel R, Rodriguez C, Feigelson HS, Bandera EV, Gansler T, Thun MJ \& Calle EE 2008 Body mass and endometrial cancer risk by hormone replacement therapy and cancer subtype. Cancer Epidemiology Biomarkers and Prevention 17 73-79. (doi:10.1158/1055-9965.EPI-07-2567)

4 Li XF \& Ahmed A 1996 Expression of angiotensin II and its receptor subtypes in endometrial hyperplasia: a possible role in dysfunctional menstruation. Laboratory Investigation 75 137-145.

5 Li XF \& Ahmed A 1997 Compartmentalization and cyclic variation of immunoreactivity of renin and angiotensin converting enzyme in human endometrium throughout the menstrual cycle. Human Reproduction 12 2804-2809. (doi:10.1093/humrep/12.12.2804)

6 Pringle KG, Tadros MA, Callister RJ \& Lumbers ER 2011 The expression and localization of the human placental prorenin/reninangiotensin system throughout pregnancy: roles in trophoblast invasion and angiogenesis? Placenta 32 956-962. (doi:10.1016/j. placenta.2011.09.020)

7 Nguyen G \& Muller DN 2010 The biology of the (pro)renin receptor. Journal of the American Society of Nephrology 21 18-23. (doi:10.1681/ ASN.2009030300)

8 Chandra S, Narang R, Sreenivas V, Bhatia J, Saluja D \& Srivastava K 2014 Association of angiotensin II type 1 receptor (A1166C) gene polymorphism and its increased expression in essential hypertension: a case-control study. PLOS ONE 9 e101502. (doi:10.1371/journal. pone.0101502)

9 Pringle KG, Delforce SJ, Wang Y, Ashton KA, Proietto A, Otton G, Blackwell CC, Scott RJ \& Lumbers ER 2016 Renin-angiotensin system gene polymorphisms and endometrial cancer. Endocrine Connections 5 128-135. (doi:10.1530/EC-15-0112)

10 Piastowska-Ciesielska AW, Płuciennik E, Wójcik-Krowiranda K, Bieńkiewicz A, Bednarek A \& Ochędalski T 2012 Analysis of the expression of angiotensin II type 1 receptor and VEGF in endometrial adenocarcinoma with different clinicopathological characteristics. Tumor Biology 33 767-774. (doi:10.1007/s13277-011-0292-0)

11 Shibata K, Kikkawa F, Mizokami Y, Kajiyama H, Ino K, Nomura S \& Mizutani S 2005 Possible involvement of adipocyte-derived leucine aminopeptidase via angiotensin II in endometrial carcinoma. Tumor Biology 26 9-16. (doi:10.1159/000084181)

12 Morris BJ 1978 Activation of human inactive ('pro-') renin by cathepsin D and pepsin. Journal of Clinical Endocrinology and Metabolism 46 153-157. (doi:10.1210/jcem-46-1-153) 
13 Gilsbach R, Kouta M, Bonisch H \& Bruss M 2006 Comparison of in vitro and in vivo reference genes for internal standardization of realtime PCR data. Biotechniques 40 173-177. (doi:10.2144/000112052)

14 Ferguson SE, Olshen AB, Viale A, Barakat RR \& Boyd J 2005 Stratification of intermediate-risk endometrial cancer patients into groups at high risk or low risk for recurrence based on tumor gene expression profiles. Clinical Cancer Research 11 2252-2257. (doi:10.1158/1078-0432.CCR-04-1353)

15 Deschepper CF 1994 Angiotensinogen: hormonal regulation and relative importance in the generation of angiotensin II. Kidney International 46 1561-1563. (doi:10.1038/ki.1994.446)

16 Cassis LA, Police SB, Yiannikouris F \& Thatcher SE 2008 Local adipose tissue renin-angiotensin system. Current Hypertension Reports 10 93-98. (doi:10.1007/s11906-008-0019-9)

17 Lever AF, Hole DJ, Gillis CR, McCallum IR, McInnes GT, MacKinnon PL, Meredith PA, Murray LS, Reid JL \& Robertson JW 1998
Do inhibitors of angiotensin-I-converting enzyme protect against risk of cancer? Lancet 352 179-184. (doi:10.1016/S0140-6736(98)03228-0) 18 AbdAlla S, Lother H, Abdel-tawab AM \& Quitterer U 2001 The angiotensin II AT2 receptor is an AT1 receptor antagonist. Journal of Biological Chemistry 276 39721-39726. (doi:10.1074/jbc.M105253200)

19 Kostenis E, Milligan G, Christopoulos A, Sanchez-Ferrer CF, HeringerWalther S, Sexton PM, Gembardt F, Kellett E, Martini L, Vanderheyden P, et al. 2005 G-protein-coupled receptor Mas is a physiological antagonist of the angiotensin II type 1 receptor. Circulation 111 1806-1813. (doi:10.1161/01.CIR.0000160867.23556.7D)

20 Zavadil J \& Bottinger EP 2005 TGF-beta and epithelial-to-mesenchymal transitions. Oncogene 24 5764-5774. (doi:10.1038/sj.onc.1208927)

21 Huang Y, Noble NA, Zhang J, Xu C \& Border WA 2007 Reninstimulated TGF-beta1 expression is regulated by a mitogen-activated protein kinase in mesangial cells. Kidney International 72 45-52. (doi:10.1038/sj.ki.5002243)Iqui aut rerum, sinus re, niscia debit latur?

Received in final form 22 November 2016

Accepted 12 December 2016

Accepted Preprint published online 12 December 2016 http://www.endocrineconnections.org DOI: 10.1530/EC-16-0082
C) 2017 The authors Published by Bioscientifica Ltd 